\title{
Solid State Oxidation of Phenols to Quinones with Sodium Perborate on Wet Montmorillonite K10
}

\author{
Mohammed M. Hashemi*, Bagher Eftekhari-Sis, Behzad Khalili and Zahed Karimi-Jaberi \\ Department of Chemistry, Sharif University of Technology, P.O. Box 11365-9516, Tehran, Iran
}

\begin{abstract}
Fenóis foram oxidados a quinonas usando perborato de sódio (SPB) em montmorilonita úmida, como oxidante. A reação foi conduzida em fase sólida, a temperatura ambiente e sem a utilização de solventes.
\end{abstract}

Phenols were oxidized to quinones using sodium perborate (SPB) on wet montmorillonite as oxidant. The reaction was carried out at ambient temperature on the solid phase under solvent free conditions.

Keywords: oxidation, sodium perborate, phenols, quinones

\section{Introduction}

Natural products having a benzoquinone structure show biologically important properties such as cardiovascular, antitumour, antibacterial, antigerminative and antiprotozoan activities. ${ }^{1}$ 2-Methyl-1,4-naphthoquinone, vitamin $\mathrm{K} 3$, constitutes an important additive in animal feed, which is used commercially in large quantities. Furthermore, benzoquinones are important fine-chemicals in industry and are useful dienophiles in chemical transformations and synthesis of many natural products. ${ }^{2}$ Thus, trimethyl-p-benzoquinone and 2,3dimethoxy-5-methyl-p-benzo-quinone are especially valuable starting materials for the synthesis of vitamin $\mathrm{E}$ and coenzyme $\mathrm{Q}$ and therefore, are important in medicine. ${ }^{3}$ Benzoquinones are usually prepared by direct oxidation of phenol and methoxy benzene derivatives. The most commonly employed oxidants for these syntheses are Fremy's salt, ${ }^{4}$ oxygen/salcomine, ${ }^{5}$ chromic acid, ${ }^{6}$ Weitz's salt (BAHA), ${ }^{7}$ ceric ammonium nitrate (CAN), ${ }^{8}$ ortho-chloranil (tetrachlorobenzo-1,2-quinone), ${ }^{9}$ chromium (VI) salts, ${ }^{10}$ hypervalent iodine (III), ${ }^{11} \mathrm{~N}$ bromosuccinimide (NBS), ${ }^{12}$ cobalt and manganese salts in the presence of oxygen, ${ }^{13}$ hydrogen peroxide, ${ }^{14}$ and silver (I) oxide- $\mathrm{HNO}_{3}$ system. ${ }^{15}$ However, the different reagents show varying degree of success as well as limitations due to high reaction temperatures ${ }^{16}$ the possibility of explosion, ${ }^{17}$ strongly basic or acidic

*e-mail: mhashemi@ sharif.edu conditions which may be incompatible with substituents on the aromatic rings ${ }^{18}$ and most of these reagents involve metal ions and solvents that are not ecofriendly. There is increasing emphasis on technical developments that employ environmentally friendly reagents or proceed in the absence of solvent and preferably without a metal ion thereby reducing the waste generation.

Sodium perborate (SPB) is an inexpensive large scale industrial oxidant used in bleaching which is safe to handle. ${ }^{19}$ It has also been used for oxidation of various functional groups ${ }^{20}$ such as anilines to nitroarenes, ${ }^{21}$ sulfides, ${ }^{22}$ aromatic aldehydes to carboxylic acids, ${ }^{23}$ oxidative hydration of aromatic nitriles to amides, ${ }^{22}$ and asymmetric epoxidation of substituted chalcones. ${ }^{24}$ McKillop reported oxidation of phenols to quinones with SPB in acetic acid. ${ }^{21}$ Although interesting results were reported, this method shows some disadvantages like long reaction times and low yields especially for the monohydric phenols.

\section{Results and Discussion}

In connection with our interest in the oxidation of organic compounds, ${ }^{25}$ we describe here a facile oxidation of phenols to quinones in solid state using SPB on wet montmorillonite $\mathrm{K} 10$ as oxidant.

Different types of phenols were subjected to the oxidation by simple admixing with SPB and wet montmorillonite K10 $(80 \% \mathrm{~m} / \mathrm{m})$. The process involves grinding phenols with SPB and wet montmorillonite K10 using a mortar and pestle. After thoroughly grinding, each 
phenol develops an especial color within 1-5 minutes, which indicates the completion of the reaction, which also was monitored by TLC examination.

Supported SPB on wet montmorillonite K10 is an excellent reagent for oxidation of phenols to quinones as shown in Scheme 1. Ortho-quinones are not produced by this reagent except when ortho-aminophenol and orthohydroxyphenol are used. In most cases the optimum mole ratio between phenol and SPB was found to be 1:2 which gave the best results.

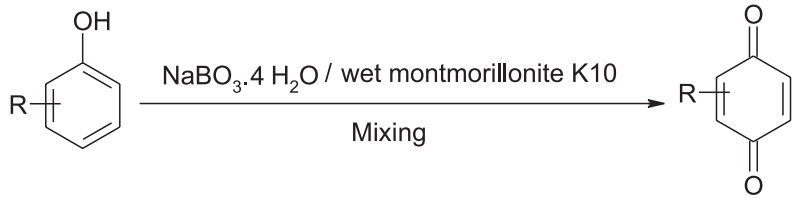

Scheme 1.

Table 1. Oxidation of phenols to quinones using $\mathrm{SPB}^{\mathrm{a}}$

\begin{tabular}{lcccc}
\hline Entry & Substrate & \multicolumn{3}{c}{ Yield\% } \\
\cline { 2 - 5 } & $\begin{array}{c}\text { wet } \\
\text { montmorillonite K10 }\end{array}$ & $\begin{array}{c}\text { wet } \\
\text { silica gel }\end{array}$ & $\begin{array}{c}\text { wet } \\
\text { alumina }\end{array}$ \\
\hline 1 & hydroquinone & 93 & 69 & 73 \\
2 & 2,6-dimethylphenol & 65 & 53 & 51 \\
\hline
\end{tabular}

${ }^{a}$ All yields refer to isolated products.

As shown in Table 1 montmorillonite K10 was found to be the best surface for the oxidation of phenols to quinones with SPB.

The results of oxidation of phenols to quinones with SPB on wet montmorillonite K10 are summarized in Table 2. All yields refer to isolated products.

\section{Conclusions}

In summary we have extended successfully the application of SPB on wet montmorillonite K10 for the oxidation of phenols to quinones. This method offers some advantages in terms of simplicity of performance, solvent free conditions, ambient temperature, high yields and short reaction times.

\section{Experimental}

All products are known compounds and were characterized by comparison of their physical and spectroscopic data with those of authentic samples. ${ }^{1} \mathrm{H}$ NMR spectra were recorded on Bruker DPX500 spectrometer in $\mathrm{CDCl}_{3}$ containing TMS as in internal standard and IR spectra were recorded on Perkin Elmer 781 spectrometer.

\section{General procedure for the oxidation of phenols to quinones}

SPB (2 mmol) and wet montmorillonite K10 (1 g, 80\% $\mathrm{m} / \mathrm{m}$ ) were mixed thoroughly. Then phenol (1 mmol) was added and mixed thoroughly using a mortar and pestle. After the completion of the reaction (TLC; petroleum ether/ethyl acetate 4:1), the product was extracted with chloroform, washed with $5 \% \mathrm{NaOH}$ aqueous solution and dried over $\mathrm{Na}_{2} \mathrm{SO}_{4}$. The solvent was evaporated and the product purified by short column chromatography over silica gel (petroleum ether/ethyl acetate 4:1).

1,4-Benzoquinone. Yellow solid, mp $114-116{ }^{\circ} \mathrm{C}$ (lit. ${ }^{26}$ 115-117 $\left.{ }^{\circ} \mathrm{C}\right)$; IR $v_{\max } / \mathrm{cm}^{-1}: 3060,1680,1658,1600,1570$, $1510(\mathrm{KBr}) ;{ }^{1} \mathrm{H} \mathrm{NMR}\left(\mathrm{CDCl}_{3}, 500 \mathrm{MHz}\right) \delta 6.8$ (s, 4H).

2-Methyl-1,4-benzoquinone. Yellow-purple solid, mp 69-71 ${ }^{\circ} \mathrm{C}$ (lit. ${ }^{26} 68-70{ }^{\circ} \mathrm{C}$ ); IR $v_{\max } / \mathrm{cm}^{-1}: 3030,1653$, $1345,1100,930(\mathrm{KBr}) ;{ }^{1} \mathrm{H} \mathrm{NMR}\left(\mathrm{CDCl}_{3}, 500 \mathrm{MHz}\right) \delta 6.6-$ $6.7(\mathrm{~m}, 3 \mathrm{H}), 2.0(\mathrm{~s}, 3 \mathrm{H})$.

2,6-Dimethyl-1,4-benzoquinone. Yellow solid, mp 71-73 ${ }^{\circ} \mathrm{C}$ (lit. ${ }^{26} 72{ }^{\circ} \mathrm{C}$ ); IR $v_{\max } / \mathrm{cm}^{-1}: 3030,1648,1300,1100,930$ $(\mathrm{KBr}) ;{ }^{1} \mathrm{H} \mathrm{NMR}\left(\mathrm{CDCl}_{3}, 500 \mathrm{MHz}\right) \delta 6.7(\mathrm{~s}, 2 \mathrm{H}), 1.9(\mathrm{~s}, 6 \mathrm{H})$.

Table 2. Oxidation of phenols to quinones using SPB/wet montmorillonite K10

\begin{tabular}{|c|c|c|c|c|}
\hline Entry & Substrate & Product & time $(\min )$ & $(\mathrm{m} / \mathrm{g})$ Yield $(\%)^{\mathrm{a}}$ \\
\hline 1 & phenol & 1,4-benzoquinone & 5 & $(0.06) 56$ \\
\hline 2 & hydroquinone & 1,4-benzoquinone & 1 & (0.10) 93 \\
\hline 3 & 2-methylphenol & 2-methyl-1,4-benzoquinone & 5 & $(0.08) 61$ \\
\hline 4 & 3-methylphenol & 2-methyl-1,4-benzoquinone & 5 & $(0.07) 57$ \\
\hline 5 & 2,6-dimethylphenol & 2,6-dimethyl-1,4-benzoquinone & 1 & (0.09) 65 \\
\hline 6 & 4-aminophenol & 1,4-benzoquinone & 3 & $(0.09) 81$ \\
\hline 7 & 2-hydroxyphenol & 1,2-benzoquinone & 4 & $(0.07) 64$ \\
\hline 8 & 2-aminophenol & 1,2-benzoquinone & 4 & $(0.07) 66$ \\
\hline 9 & 1-naphthol & 1,4-naphthoquinone & 5 & $(0.10) 63$ \\
\hline 10 & 1,4-dihydroxy naphthalene & 1,4-naphthoquinone & 2 & $(0.11) 73$ \\
\hline 11 & 2-chlorophenol & 2-chloro-1,4-benzoquinone & 5 & (0.07) 47 \\
\hline
\end{tabular}

${ }^{\mathrm{a}} \mathrm{All}$ yields refer to isolated products. 
1,2-Benzoquinone. Red-purple solid, decomposed at $61{ }^{\circ} \mathrm{C}$ (lit. ${ }^{26} 60-70{ }^{\circ} \mathrm{C}$ ); IR $v_{\max } / \mathrm{cm}^{-1}: 3050,2910,1748$, 1688, 1670, 1410, 1292 (KBr).

1,4-Naphthoquinone. Yellow solid, mp $127^{\circ} \mathrm{C}$ (lit. ${ }^{26}$ $\left.128.5^{\circ} \mathrm{C}\right) ; \mathrm{IR} v_{\max } / \mathrm{cm}^{-1}: 3030,1675,1610,1590,1485$, 1330, $1320(\mathrm{KBr}) ;{ }^{1} \mathrm{H} \mathrm{NMR}\left(\mathrm{CDCl}_{3}, 500 \mathrm{MHz}\right) \delta$ 7.7-8.2 (m, 4H), 7.0 (s, 2H).

2-Chloro-1,4-benzoquinone. Yellow-red solid, mp 55$57^{\circ} \mathrm{C}$ (lit. $\left.{ }^{26} 57{ }^{\circ} \mathrm{C}\right) ; \mathrm{IR} v_{\max } / \mathrm{cm}^{-1}: 3050,1691,1662,1590$, $1370,1330,1290(\mathrm{KBr}) ;{ }^{\prime} \mathrm{H} \mathrm{NMR}\left(\mathrm{CDCl}_{3}, 500 \mathrm{MHz}\right) \delta 6.9$ (s, 1H), 6.4-6.8 (m, 2H).

\section{References}

1. Nishina, A.; Uchibori, T.; Agric. Biol. Chem. 1991, 55, 2395; Letcher, R. M.; Wong, M. -C.; J. Chem. Soc. Perkin Trans. 1 1992, 3035; Floreani, M.; Forlin, A.; Bellin, S.; Carpanedo, F.; Gen. Pharmacol. 1996, 27, 873; Miles, D. H.; Payne, M.; Tetrahedron 2001, 57, 5769.

2. Quideau, S.; Pouységu, L.; Org. Prep. Proced. Int. 1999, 31, 617, and references cited therein.

3. Yamada, S.; Takeshita, T.; Tanaka, J.; J. Synth. Org. Chem. Jpn. 1982, 40, 268.

4. Chercaoui, O.; Nebois, P.; Fillion, H.; Domard, M.; Fenet, B.; Tetrahedron 1996, 52, 9499; Itoh, S.; Ogino, M.; Haranou, S.; Terasaka, T.; Ando, T.; Komatsu, M.; Ohshiro, Y.; Fukuzumi, S.; Kano, K.; Takagi, K.; Ikeda, T.; J. Am. Chem. Soc. 1995, 117, 1485.

5. Dockal, E. R.; Cass, Q. B.; Brocksom, T. J.; Brocksom, U.; Corrêa, A. G.; Synth. Commun. 1985, 15, 1033.

6. Liotta, D.; Arbiser, J.; Short, J. W.; Saindane, M.; J. Org. Chem. 1983, 48, 2932.

7. Takeya, T.; Moregi, S.; Itoh, T.; Tobinaga, S.; Chem. Pharm. Bull. 1997, 45, 613.

8. Brimble, M. A.; Duncalf, L. J.; Phythian, S. J.; J. Chem. Soc. Perkin. Trans. 1 1997, 1399.

9. Feldman, K. S.; Quideau, S.; Appel, H. M.; J. Org. Chem. 1996, 61, 6656.
10. Vanderlei, J. M. L.; Coelho, F.; Almeida, W. P.; Tetrahedron: Asymmetry 1997, 8, 2781; Almeida, W. P.; Correia, C. R. D.; Tetrahedron Lett. 1994, 35, 1367.

11. Kinugawa, M.; Masuda, Y.; Arai, H.; Nishikawa, H.; Osaga, T.; Tomioka, S.; Kasai, M.; Synthesis 1996, 633; Kato, N.; Sugaya, T.; Mimura, T.; Ikuta, M.; Kato, S.; Kuge, Y.; Tomioka, S.; Kasai, M.; Synthesis 1997, 625.

12. Boyd, V. A.; Sulikowski, G. A.; J. Am. Chem. Soc. 1995, 117, 8472 .

13. Hashemi, M. M.; Beni, Y. A.; J. Chem. Res. (S) 1998, 138.

14. Saladino, R.; Neri, V.; Mincione, E.; Filippone, P.; Tetrahedron 2002, 58, 8493.

15. Brimble, M. A.; Phythian, S. J.; Tetrahedron Lett. 1993, 34, 5813.

16. Shishido, K.; Goto, K.; Miyoshi, S.; Takaishi, Y.; Shibuya, M.; J. Org. Chem. 1994, 59, 406.

17. Moser, W.; Howie, R. A.; J. Chem. Soc. A: Inorg. Phys. Theor. 1968, 3039

18. Watanabe, M.; Shinoda, E.; Shimizu, Y.; Furukawa, S.; Iwao, M.; Kuraishi, T.; Tetrahedron 1987, 43, 5281.

19. Kirk-Othmer; Encyclopedia of Chemical Technology, John Wiley and Sons: New York, 1987.

20. McKillop, A.; Sanderson, W. R.; Tetrahedron 1995, 51, 6145; Muzart, J.; Synthesis 1995, 1325.

21. McKillop, A.; Tarbin, J. A.; Tetrahedron 1987, 43, 1753; McKillop, A.; Tarbin, J. A.; Tetrahedron Lett. 1983, 24, 1505.

22. Aggarwal, V. K.; Davies, I. W.; Franklin, R.; Maddock, J.; Mahon, M. F.; Molloy, K. C.; J. Chem. Soc. Perkin Trans. 1 1994, 2363.

23. McKillop, A.; Kemp, D.; Tetrahedron 1989, 45, 3299.

24. Savizky, R. M.; Suzuki, N.; Bové, J. L.; Tetrahedron: Asymmetry 1998, 9, 3967.

25. Hashemi, M. M.; Akhbari, M.; Monatsh. Chem. 2003, 134, 1561; Hashemi, M. M.; Karimi-Jaberi, Z.; J. Chem. Res. 2004, 364; Hashemi, M. M.; Karimi-Jaberi, Z.; Monatsh. Chem. 2004, 135, 41; Hashemi, M. M.; Karimi-Jaberi, Z.; Eftekhari-Sis, B.; J. Chem. Res. 2005, 161.

26. Weast, R. C.; Grasselli, J. G.; CRC Handbook of Data on Organic Compounds, $2^{\text {nd }}$ ed., CRC Press: Boca Raton, FL, 1989. 